\title{
Thermo-mechanical and superimposed high- cycle fatigue interactions in compacted graphite iron
}

\author{
Viktor Norman, Peter Skoglund, Daniel Leidermark and Johan Moverare
}

\section{Linköping University Post Print}

\section{Tweet}

N.B.: When citing this work, cite the original article.

Original Publication:

Viktor Norman, Peter Skoglund, Daniel Leidermark and Johan Moverare, Thermo-mechanical and superimposed high-cycle fatigue interactions in compacted graphite iron, 2015, International Journal of Fatigue, (80), 381-390.

http://dx.doi.org/10.1016/j.ijfatigue.2015.06.005

Copyright: Elsevier

http://www.elsevier.com/

Postprint available at: Linköping University Electronic Press

http://urn.kb.se/resolve?urn=urn:nbn:se:liu:diva-121029 


\title{
Thermo-mechanical and superimposed high-cycle fatigue interactions in compacted graphite iron
}

\author{
V. Norman ${ }^{\mathrm{a}, *}$, P. Skoglund ${ }^{\mathrm{a}, \mathrm{c}}$, D. Leidermark ${ }^{\mathrm{b}}$, J. Moverare ${ }^{\mathrm{a}}$ \\ ${ }^{a}$ Division of Engineering Materials, Department of Management and Engineering, \\ Linköping University, SE-58183 Linköping, Sweden \\ ${ }^{b}$ Division of Solid Mechanics, Department of Management and Engineering, Linköping \\ University, SE-58183 Linköping, Sweden \\ ${ }^{c}$ Scania CV AB, Materials Technology, SE-15187 Södertälje, Sweden
}

\begin{abstract}
The effect of adding a superimposed high-frequent strain load, denoted as a high-cycle fatigue strain component, upon a strain-controlled thermomechanical fatigue test has been studied on a compacted graphite iron ENGJV-400 for different thermo-mechanical fatigue cycles and high-cycle fatigue strain ranges. It is demonstrated that the successive application of an high-cycle fatigue load has a consistent effect on the fatigue life, namely the existence of a constant high-cycle fatigue strain range threshold below which the fatigue life is unaffected but severely reduced when above. This effect on the fatigue life is predicted assuming that microstructurally small cracks are propagated and accelerated according to a Paris law incorporating an experimentally estimated crack opening level.
\end{abstract}

Keywords: Cast iron, Thermo-mechanical fatigue, High-cycle fatigue, Fatigue crack growth, Life prediction

\section{Introduction}

The cast iron family, in which compacted graphite iron (CGI) is a member, is one of the most common material groups used in diesel engine components, such as the cylinder head in the heavy-vehicle automotive industry. The reason for such a material choice is the material shaping technique used for castings, which is associated with a low cost and the convenience when producing components with a complex geometry.

After being manufactured and put into operation, the cast iron engine component is exposed to an aggressive environment involving time-varying mechanical loads and high temperatures due to the cyclic nature of the engine operation, commonly referred to as the start-operate-stop cycle [1]. During one such period of the time-dependent operation, the material is heated and cooled as the engine starts up and shuts down implying a thermal expansion and contraction. In between, the material is exposed to an elevated temperature as the engine

\footnotetext{
*Corresponding author. Phone: 004613284695

Email address: viktor.norman@liu.se (V. Norman)
} 


\begin{tabular}{|c|c|c|}
\hline \multicolumn{3}{|l|}{ Nomenclature } \\
\hline $\begin{array}{ll}\bar{a} & \text { Average microcrack size } \\
\bar{a}_{0} & \text { Initital average microcrack }\end{array}$ & $\sigma_{o p}$ & $\begin{array}{l}\text { Crack opening engineering } \\
\text { stress }\end{array}$ \\
\hline size & $\varepsilon$ & Extensometer strain \\
\hline $\begin{array}{l}\text { Final average microcrack } \\
\text { size }\end{array}$ & $\varepsilon_{H C F}$ & High-cycle fatigue strain \\
\hline Geometric constant & $\varepsilon_{\text {Mech }}$ & Total mechanical strain \\
\hline Incremental total crack ex- & $\varepsilon_{T h}$ & Thermal strain \\
\hline tension & $\varepsilon_{T M F}$ & Thermo-mechanical strain \\
\hline $\begin{array}{c}\Delta a_{H C F} \text { Incremental crack exten- } \\
\text { sion due to a HCF cycle }\end{array}$ & $A$ & $\begin{array}{l}\text { Percent elongation after frac- } \\
\text { ture }\end{array}$ \\
\hline$\Delta a_{T M F}$ Incremental crack exten- & $C$ & Paris law coefficient \\
\hline sion due to a TMF cycle & $C^{\prime}$ & Fitting parameter \\
\hline $\begin{array}{ll}\Delta K & \text { Stress-intensity factor range } \\
\Delta N & \text { Number of cycles increment }\end{array}$ & $C_{H C F}$ & HCF cycle Paris law coeffi- \\
\hline $\begin{array}{l}\Delta \sigma \quad \text { Engineering stress range } \\
\Delta \sigma_{H C F}^{E f f} \quad \text { Effective } \mathrm{HCF} \text { engineering }\end{array}$ & $C_{T M F}$ & $\begin{array}{l}\text { TMF cycle Paris law coeffi- } \\
\text { cient }\end{array}$ \\
\hline stress range & $E$ & Elastic modulus \\
\hline$\Delta \varepsilon_{H C F}$ HCF strain range & $I$ & Constant \\
\hline$\Delta \varepsilon_{M e c h}$ Total mechanical strain & $N$ & Number of cycles \\
\hline range & $n$ & Paris law exponent \\
\hline$\Delta \varepsilon_{T M F}$ TMF strain range & $N_{f}$ & Number of cycles to failure \\
\hline $\begin{array}{ll}\frac{d \bar{a}}{d N} & \begin{array}{l}\text { Avarage } \\
\text { rate }\end{array}\end{array}$ & $n_{H C F}$ & $\begin{array}{l}\text { HCF cycle Paris law expo- } \\
\text { nent }\end{array}$ \\
\hline $\begin{array}{l}\text { Number of HCF cycles above } \\
\text { the crack opening engineer- }\end{array}$ & $n_{T M F}$ & $\begin{array}{l}\text { TMF cycle Paris law expo- } \\
\text { nent }\end{array}$ \\
\hline & $R_{m}$ & Tensile strength \\
\hline Engineering stress & $R_{p 0.02 \%}$ & $0.02 \%$ off-set yield strength \\
\hline$\sigma_{\text {on }}$ HCF cycle on/off engineer- & $T_{\operatorname{Max}}$ & Maximum temperature \\
\hline ing stress & $T_{\text {Min }}$ & Minimum temperature \\
\hline
\end{tabular}

operates at its service temperature, presumably found in the vicinity of $400^{\circ} \mathrm{C}$, during which relaxation and oxidation processes occur. On top of this, there are superimposed high-frequent vibrations caused by the rotational motion in the engine and thus present over the whole operation cycle. Inevitably, this complex load condition will promote the fatigue damage propagation which eventually will result in the significant degradation of the material, thereby rendering the engine beyond operation capability. 
These life-limiting aspects can be alleviated by a sophisticated engine design which to a wider extent is based on computer simulations using commercial finite element (FE) software rather than on component prototype testing $[1,2,3,4]$. Such simulations are often divided into three uncoupled analyses, the thermal, mechanical and fatigue analysis [2], where the development of the latter is the subject of this paper. To construct such an analysis, which relates an estimate of the lifetime to given values of the thermal and mechanical loads, extensive experimental information is required upon which the fatigue model is based. This could be low-cycle fatigue (LCF) or crack propagation data collected from laboratory tests, however lately, thermo-mechanical fatigue (TMF) testing has become one of the most commonly employed sources of fatigue data. These tests consist of uniaxial fatigue testing with a simultaneous change in the temperature and mechanical strain, which in this way incorporates the aspect of the thermal variation seen in the real applications.

Recently, attention has also been given to the combined effect of TMF and superimposed high-frequent strain loads, commonly referred to as superimposed high-cycle fatigue (HCF), which simulates the vibrations present during the engine operation [5]. It has been identified that such an additional load might have a significant impact on the total life of the component, even at strain ranges far below the endurance limit, with examples ranging from cast aluminium and superalloys to cast irons $[6,7,8,9]$. Therefore, it has become evident that such vibrations can no longer be neglected as it has been done in the past.

The main purpose of this paper is to quantify and predict the TMF fatigue life reduction due to a superimposed HCF strain load on a CGI. Such experimental data has already been reported for a similar type of material [7], however not extensively enough in order to draw wide conclusions or to fit a predictive model. Furthermore, an improved test approach has been employed different from what previously reported in the literature regarding TMF-HCF testing. The test approach allows an intuitive presentation and clear interpretation of the interaction between these two fatigue modes. The obtained experimental results and the proposed prediction model will be of great industrial use in the design of heavy-vehicle automotive components as they will further clarify and quantify the importance of superimposed high-frequent strain loading.

\section{Background}

\subsection{Thermo-mechanical fatigue}

Thermo-mechanical fatigue (TMF) testing, in contrast to conventional isothermal low-cycle fatigue (LCF) tests, involves both a cyclic mechanical load, either stress or strain controlled, and a cyclic temperature variation $[10,11]$. The thermal and mechanical cycle periods are most commonly the same, however tests are often varied by using different phase shifts between the two. The two extreme cases commonly applied in testing are 0 and 180 degree phase shift which are known as an in-phase (IP) and out-of-phase (OP) configuration respectively. In the latter, the maximum temperature coincides with the minimum strain value, which could be negative, and vice versa for the former.

The instantaneous uniaxial strain $\varepsilon(t)$ applied during a TMF test is composed of two components, the mechanical strain $\varepsilon_{M e c h}(t)$ which is due to the applied stress $\sigma(t)$, and the thermal strain $\varepsilon_{T h}(t)$ representing the thermal expansion. 


$$
\varepsilon(t)=\varepsilon_{M e c h}(t)+\varepsilon_{T h}(t)
$$

For instance, if a temperature cycle is applied while simultaneously compensating the thermal expansion $\varepsilon_{T h}$ with a mechanical strain $\varepsilon_{M e c h}$ of the same magnitude but of the opposite sign, the total strain $\varepsilon$ will be zero and the test will be in an OP configuration. In this case, the specimen will be denoted as rigidly clamped since no net deformation will be observed.

In the application of diesel engine cylinder heads, the characteristic loading is associated with the OP configuration [3] since the thermal expansion of hot parts is impeded by the surrounding unheated material. In particular, the valve bridges between the inlets and outlets are the most exposed area to such loading. With this in mind, TMF tests are constructed to simulate these loading conditions on simple laboratory specimens using the same range of temperatures and mechanical strains. Thus, the results obtained in this way should be more suitable to use for fatigue life prediction than for instance LCF lifetimes since the former tests will capture the actual fatigue mechanisms of the application.

\subsection{Superimposed high-cycle fatigue}

This paper takes an interest in the application of superimposed high-cycle fatigue (HCF) strain load upon a TMF test to see the effect on the fatigue life, i.e. the number of cycles to failure. Thus, an high-frequent strain oscillation is applied around the mechanical strain signal with a significantly shorter period than the overall cycle period, see Figure 1.

To make a clear distinction of all the strain components the mechanical strain signal, around which the HCF strain is oscillating, is referred to as the thermo-mechanical fatigue strain $\left(\varepsilon_{T M F}\right)$ while the combined strain signal will be referred to as the total mechanical strain $\left(\varepsilon_{M e c h}\right)$. Consequently, the HCF strain $\left(\varepsilon_{H C F}\right)$ is defined as the strain deviated from the TMF strain. Thus, the instantaneous strain given by Equation 1 is decomposed into:

$$
\varepsilon(t)=\varepsilon_{T M F}(t)+\varepsilon_{H C F}(t)+\varepsilon_{T h}(t)
$$

Moreover, the definition of the $\operatorname{HCF}\left(\Delta \varepsilon_{H C F}\right)$, TMF $\left(\Delta \varepsilon_{T M F}\right)$ and total mechanical strain range $\left(\Delta \varepsilon_{\text {Mech }}\right)$ follow accordingly, see Figure 1b. Evidently, the TMF and the total mechanical strain range coincide when the HCF strain range is zero.

There are however some aspects that need to be clarified when a comparison between TMF and TMF-HCF tests is to be performed in an OP configuration, i.e. to be able to see the difference when superimposing an HCF strain range $\left(\Delta \varepsilon_{H C F}\right)$ to a TMF test. Intuitively, the total mechanical strain range $\left(\Delta \varepsilon_{M e c h}\right)$ should be kept constant as $\Delta \varepsilon_{H C F}$ is increased, because otherwise there would be two effects on the fatigue life; reduction due to an increased $\Delta \varepsilon_{M e c h}$ and due to the occurrence of an HCF strain range. This requirement necessitates a reduction of the TMF strain range $\left(\Delta \varepsilon_{T M F}\right)$ as $\Delta \varepsilon_{H C F}$ is increased, which in principle can be achieved in two ways.

Either (i) the minimum (or maximum) value of the TMF strain is kept fixed as $\Delta \varepsilon_{T M F}$ is decreased and $\Delta \varepsilon_{H C F}$ is increased, leading to a downward (upward) shift of the minimum and maximum values of the total mechanical 


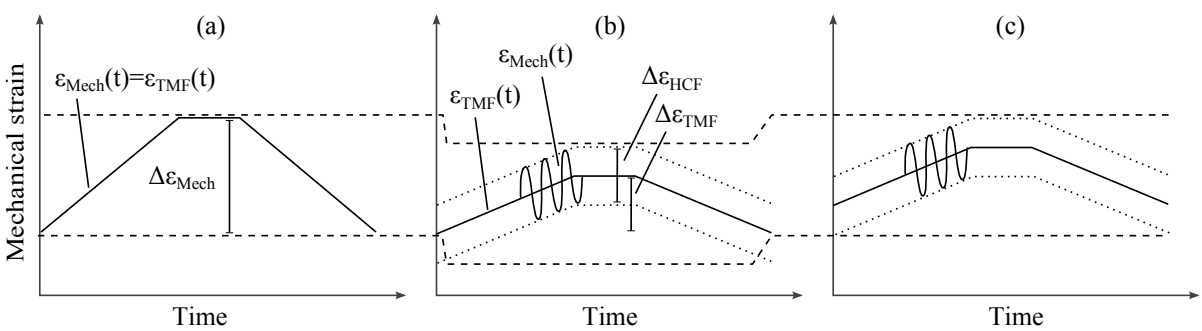

Figure 1: A schematic illustration of the mechanical strain signal applied in a regular TMF test (a) and a TMF-HCF test (b),(c). The dotted lines represent the limit values within which the HCF strain component $\varepsilon_{H C F}(t)$ is varying while the solid line represent the TMF strain $\varepsilon_{T M F}(t)$. Figure (b) corresponds to the (i) case while (c) corresponds to (ii).

strain, see Figure 1b. Alternatively, (ii) the minimum and maximum value of the total mechanical strain are kept fixed, which instead implies an upward shift of the TMF strain signal, see Figure 1c.

In this study, the (ii) approach shown in Figure 1c has been chosen when varying the HCF strain range since it is argued that this method yields a better justified comparison of TMF and TMF-HCF tests, however the (i) approach as shown in Figure $1 \mathrm{~b}$ is commonly seen in literature [6, 8, 12, 7]. As seen in Figure $1 \mathrm{~b}$, the (i) case will result in a different $R_{\varepsilon}$-value which will depend on the value of $\Delta \varepsilon_{H C F}$. This is possibly a problem since the fatigue life could be dependent on the $R_{\varepsilon}$-value. In addition, the hysteresis loops of a TMF test and a TMF-HCF test will have similar envelopes for the (ii) case, see Figure $7 \mathrm{~b}$ in the result section, i.e. the only significant difference in the TMF-HCF test is a high-frequent partial unloading from the hysteresis envelope, while the position of the envelope almost overlaps with the hysteresis loop of the TMF test. This is not true for the (i) case, instead the TMF-HCF hysteresis loop will be shifted to the right due to the upward shift of the minimum and maximum value of the total mechanical strain mentioned above. Since this study aims to investigate the separated influence of the superimposed HCF strain load, the (ii) test configuration is thus argued to be the most suitable choice.

Uihlein et al. [7] studied the interaction between TMF and superimposed $\mathrm{HCF}$ for three different cast irons, namely EN-GJS-700, EN-GJV-450 and ENGJL-250, i.e. a spheroidal (SGI), compacted (CGI) and lamellar graphite iron (LGI). They varied the HCF strain range according to the (i) method which led to the observation of an HCF strain range threshold beneath which the fatigue life of all three cast irons was only insignificantly affected while severely shortened when above. From the test results of the CGI they could estimate the $\mathrm{HCF}$ strain range threshold to $0.06 \%$ in this material.

On the related SGI material, EN-GJS-700, together with several other materials, Beck et al. [8] made a similar investigation using the (i) method and found that the SGI was the only investigated material without an HCF strain range threshold. In addition, they observed that the application of an HCF strain load did not induce new cracks nor did it change the fatigue crack mechanism, rather it only accelerated the growth of the already existing cracks. Thus, it is suggested in this study that this behaviour could also be true for CGI, i.e. that the crack growth due to the HCF strain can be superimposed to the TMF crack propagation, since the material is similar to SGI. 


\section{Material and experimental procedure}

\subsection{Material and specimens}

The tested material is a fully pearlitic compacted graphite iron (CGI), ENGJV-400, which is a commonly used material in cylinder heads. The chemical composition of the investigated batch is given in Table 1 . The mechanical test specimens were cut from the inside of a $16 \mathrm{~mm}$ thick cast plate, i.e. from regions solidified following a similar cooling curve, and afterwards machined into a number of cylindrical specimen.

Figure 2a shows the typical microstructure and an estimate of the nodularity was obtained as $10.0 \%$ using the image process software Axiovision. The material was also chemically etched using an etching agent based on picric acid and sodium hydroxide in order to measure the characteristic eutectic cell size. An average of the three largest cells measured at three different locations was determined to $293 \mu \mathrm{m}$. An example image of the etched surface is shown in Figure 2b.

\subsection{Static tests}

Tensile tests were conducted at room and elevated temperatures, namely one test at $22^{\circ} \mathrm{C}, 100^{\circ} \mathrm{C}, 400^{\circ} \mathrm{C}, 450^{\circ} \mathrm{C}$ and $500^{\circ} \mathrm{C}$, using an Instron 5982 electromechanic tensile test machine with a $100 k N$ load cell. The strain rate applied was $0.02 \% s^{-1}$. The strain was measured using an external Instron $7361 \mathrm{C}$ extensometer and an Instron SF16 furnace was utilised to apply the desired temperatures. The specimens were the same as those used in the TMF testing, i.e. cylindrical with a $6.3 \mathrm{~mm}$ diameter, $25 \mathrm{~mm}$ parallel length, $12.5 \mathrm{~mm}$ extensometer gauge length and $30 \mathrm{~mm}$ transition radius. The total length of the specimen was $145 \mathrm{~mm}$ and both ends were threaded for gripping with the thread size M12.

The elastic modulus obtained from a static test was calculated as the slope of the stress-strain curve over a stress interval of $40 \mathrm{MPa}$ to $80 \mathrm{MPa}$. This value was also used in the calculation of the $0.02 \%$ offset yield strength and the percent elongation after fracture. The latter is obtained by subtracting the elastic component of the elongation measured at fracture.

\subsection{Thermo-mechanical fatigue tests}

All the thermo-mechanical fatigue (TMF) tests were carried out in straincontrol according to a validated code-of practice [11] using an Instron 8801 servo hydraulic test machine. The specimen was heated and cooled through induction heating in combination with convection cooling by compressed air distributed onto the specimen through three nozzles. The specimen geometry was the same as the one described in section 3.2 and was in accordance with the requirements specified in the code of practice [11].

The TMF cycle consisted of a $200 \mathrm{~s}$ ramp up and down in temperature between $100^{\circ} \mathrm{C}\left(T_{M i n}\right)$ and a selected maximum temperature $\left(T_{\text {Max }}\right)$, as well as

\begin{tabular}{|c|c|c|c|c|c|c|c|c|c|}
\hline$C$ & $S i$ & $M n$ & $S$ & $P$ & $N i$ & $C u$ & $S n$ & $T i$ & $F e$ \\
\hline 3.38 & 1.90 & 0.010 & 0.374 & 0.019 & $<0.050$ & 0.97 & 0.09 & 0.011 & bal. \\
\hline
\end{tabular}

Table 1: The measured chemical composition in weight percent of EN-GJV-400. 


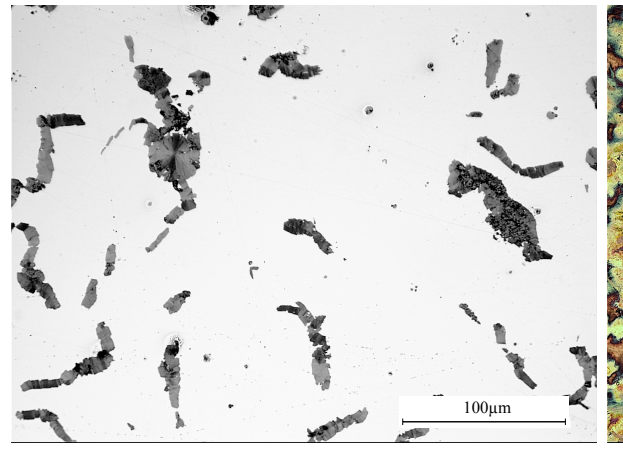

(a)

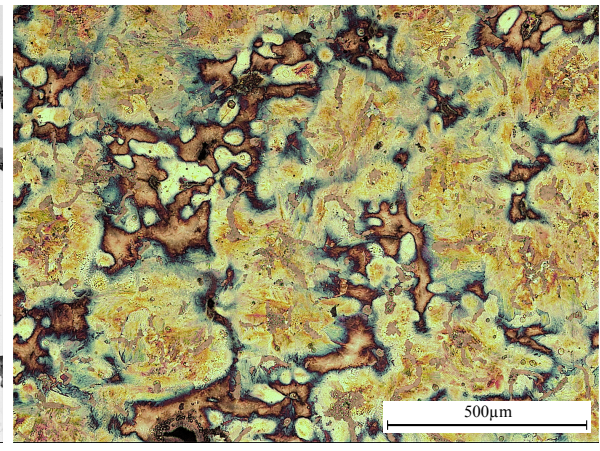

(b)

Figure 2: Examples of the characteristic (a) unetched and (b) etched microstructure.

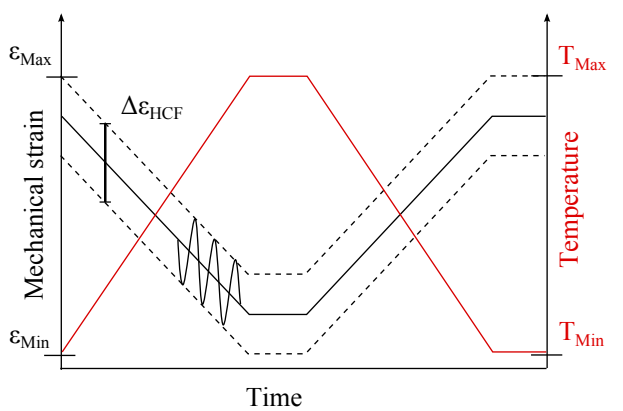

Figure 3: A schematic illustration of the temperature and mechanical strain cycles applied.

$25 s$ of dwell time at each turning point. Thus, the total cycle time was fixed to $450 \mathrm{~s}$. In combination, the total strain $(\varepsilon)$ was measured using an Instron strain gauge and thereby mechanical strain controlled using a dedicated TMF software developed by Instron. The HCF strain was conveniently applied using this software and the frequency was chosen as $15 \mathrm{~Hz}$. The actual HCF strain signal was also verified to be in good agreement with the target signal. All tests were carried out in a strain-controlled out-of-phase (OP) configuration with the maximum total mechanical strain $\left(\varepsilon_{\text {Max }}\right)$ at zero, i.e. all the test were performed with a $R_{\varepsilon}$-value of $-\infty$. Thus the studied variables were $T_{\text {Max }}, \varepsilon_{\text {Min }}$ and $\Delta \varepsilon_{H C F}$. A schematic example of the total mechanical strain and temperature cycles is shown in Figure 3. Again, note that the total mechanical strain is defined to include the HCF strain, thus when an HCF strain range is superimposed to a TMF test, the total mechanical strain range is kept constant according to Figure 1a and 1c.

It is important to note that the previously described test cycle is highly accelerated compared to the real start-operate-stop time, which typically is significantly longer than $450 \mathrm{~s}$. However, the chosen, shorter, cycle period decreases the experimental time and is also comparable to some of the component tests regularly performed on cylinder heads. Further, the investigated temperature ranges as well as the mechanical loads are also in the vicinity of the conditions found in such accelerated component tests.

All TMF tests followed the same pre-test procedure. At first, the tempera- 
ture of the specimen was cycled while keeping zero load in order to thermally stabilise the set-up and measure the thermal strain of the specimen corresponding to the chosen TMF cycle. This thermal strain information was then used to calculate the total strain $(\varepsilon)$ to be applied in order to have the desired total mechanical strain $\left(\varepsilon_{M e c h}\right)$ according to Equation 1. Subsequently, the thermal strain was verified and if approved, the test was started.

A failure criterion was also chosen in accordance with the code-of-practice [11], as a $10 \%$ drop in the stress range from a tangent line drawn at the last point of zero curvature in the stress range versus number of cycles plot.

\section{Result and Discussion}

\subsection{Static mechanical properties}

The results of the static tests are given in Table 2 and the temperature dependences of the elastic modulus and the $0.02 \%$ off-set yield strength are illustrated in Figure 4. Both the elastic modulus and the $0.02 \%$ off-set yield strength are temperature dependent within the tested temperature interval. The $0.02 \%$ off-set yield strength decreases continuously with temperature while the elastic modulus is fairly constant for low temperatures but drops significantly above $400^{\circ} \mathrm{C}$. In contrast, the percent elongation after fracture $A$ is observed to be fairly insensitive to the temperature apart from a small decrease with increasing temperature, see Table 2.

\subsection{Stress evolution during thermo-mechanical fatigue}

Figure 5a reports the evolution of the maximum stress and the stress range as the number of cycles is increased for a couple of different thermo-mechanical fatigue (TMF) tests. As a general trend, it can be seen that the maximum stress increases initially as the cycles are elapsed while the stress range is fairly constant apart from a slow and smooth variation. The trend seen in the maximum stress curve is believed to originate from the two hold times located at each turning point, i.e. at the instant of maximum and minimum temperature, see Figure 3. Because of the great difference in temperature applied at the two hold times, there will be a significant difference in the stress relaxation during the compressive hold time compared to the tensile hold time. Consequently, a net upward shift of the hysteresis loop will result, as illustrated schematically in Figure 6.

Continuing with a closer inspection of the stress range in Figure 5a, one can also conclude that these curves are affected by the difference in stress relaxation in tension and compression. The first remark is that the stress range is larger than it would have been if the test was without hold times since the upward

\begin{tabular}{|c|c|c|c|c|}
\hline$T\left[{ }^{\circ} \mathrm{C}\right]$ & $E[G P a]$ & $R_{p 0.02 \%}[M P a]$ & $R_{m}[M P a]$ & $A[\%]$ \\
\hline 22 & 154 & 252 & 476 & 2.50 \\
100 & 148 & 248 & 474 & 2.49 \\
400 & 147 & 202 & 404 & 2.49 \\
450 & 139 & 199 & 370 & 2.47 \\
500 & 92 & 187 & 322 & 2.36 \\
\hline
\end{tabular}

Table 2: Measured mechanical properties of EN-GJV-400. 


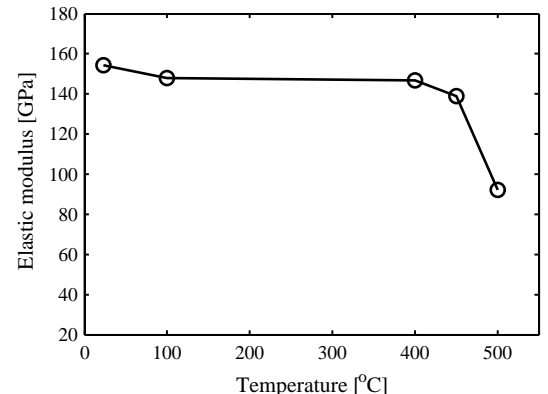

(a)

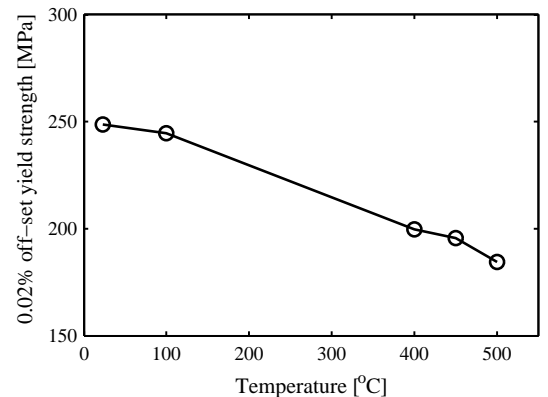

(b)

Figure 4: The temperature dependence of (a) the elastic modulus and (b) the $0.02 \%$ off-set yield strength.

shift will induce an increase of the apparent stress range, see Figure 6 . Secondly, since the total amount of stress relaxation during each compressive hold time decreases for each successive hold time, as demonstrated in Figure 5b, the contribution to the stress range decreases until the stress relaxation reaches a stable value. This explains the apparent drop during the first 10 to 20 cycles for the two tests with $T_{\operatorname{Max}}$ of $500^{\circ} \mathrm{C}$. In the curve when $T_{M a x}$ is $400^{\circ} \mathrm{C}$, this effect is believed to be small compared to the significant cyclic strain hardening. Accordingly, the absence of strain hardening in the curves when $T_{\text {Max }}$ is $500^{\circ} \mathrm{C}$ is suggested to be a result of the high temperature which probably cancels the hardening effect.

Figure 7a compares the maximum stress and the stress range for a TMF-HCF test, $100^{\circ} \mathrm{C}$ to $500^{\circ} \mathrm{C}$ and $\Delta \varepsilon_{M e c h}$ equal to $0.58 \%$, but with different applied HCF strain ranges $\left(\Delta \varepsilon_{H C F}\right)$ while keeping $\Delta \varepsilon_{M e c h}$ constant. The effect is clearly demonstrated, higher $\Delta \varepsilon_{H C F}$ results in a higher stress range while the maximum stress behaves similarly regardless of the level of $\Delta \varepsilon_{H C F}$. Thus, the minimum stress goes deeper in compression as $\Delta \varepsilon_{H C F}$ is increased, i.e. leaving the maximum stress unchanged while widening the stress range.

Figure $7 \mathrm{~b}$ shows an example of two hysteresis loops of the second cycle, one taken from a pure TMF test where $\Delta \varepsilon_{H C F}$ is zero and the other from a TMF-HCF test with $\Delta \varepsilon_{H C F}$ equal to $0.08 \%$. The envelopes of the two are quite similar, apart from that the latter obtains notably larger compressive stresses, as already observed from Figure 7a. This is believed to be due to a suppression of the recovery processes caused by the HCF oscillation, resulting in a reduction in the stress relaxation during the hot part of the cycle and consequently a higher maximum compressive stress value.

\subsection{Lifetime curves}

Figure $8 \mathrm{a}$ shows the relationship between the total mechanical strain range $\left(\Delta \varepsilon_{\text {Mech }}\right)$ and the number of cycles to failure $\left(N_{f}\right)$ for all TMF tests performed without a superimposed HCF strain range $\left(\Delta \varepsilon_{H C F}\right)$. The effect of changing $T_{M a x}$ and $\Delta \varepsilon_{M e c h}$ on the fatigue life is clearly demonstrated. The maximum temperature has a strong reductive effect as the total mechanical strain range is kept unchanged, reducing the life with a factor of 2 to 4 . 


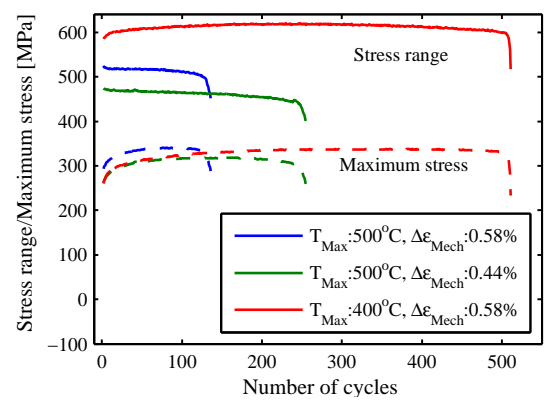

(a)

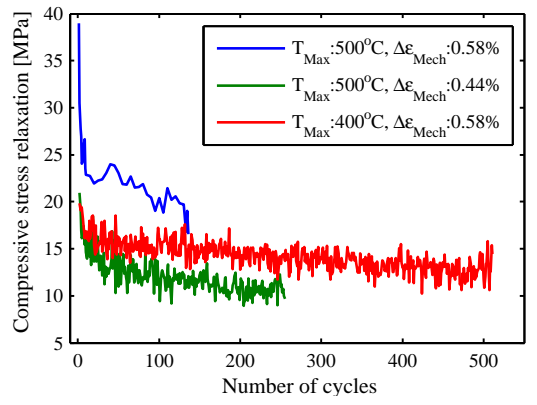

(b)

Figure 5: (a) The maximum stress and the stress range of each cycle during different TMF tests without HCF loading and (b) the accumulated stress relaxation during each compressive hold time.

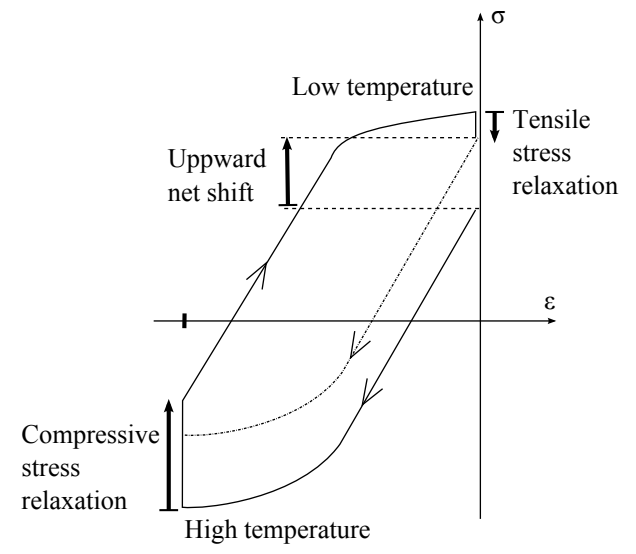

Figure 6: A schematic illustration of the evolution of the hysteresis cycle. The significant stress relaxation during the compressive hold time leads to an upward shift of the loop and a concealed increase in the stress range.

In Figure 8b the effect on $N_{f}$ when increasing $\Delta \varepsilon_{H C F}$ while maintaining a fixed temperature cycle $\left(100^{\circ} \mathrm{C}\right.$ to $\left.500^{\circ} \mathrm{C}\right)$ is shown as a conventional strain life curve. A much more convenient representation of this trend is given in Figure 9a where the horizontal axis corresponds to $\Delta \varepsilon_{H C F}$ and the vertical axis to $N_{f}$. Thus, the synergetic effect when applying different $\Delta \varepsilon_{H C F}$ values on a chosen TMF cycle is easily interpreted. Figure 9 a shows the the fatigue life dependence as $\Delta \varepsilon_{H C F}$ is successively increased in two different TMF cycles with the same temperature cycle but different $\Delta \varepsilon_{M e c h}$. Figure $9 \mathrm{~b}$ shows the same behaviour but for two TMF cycles with different $T_{M a x}$ and the same $\Delta \varepsilon_{M e c h}$.

It is clearly indicated in both Figure $9 \mathrm{a}$ and $9 \mathrm{~b}$ that $N_{f}$ is almost unaffected by a $\Delta \varepsilon_{H C F}$ value below $0.08 \%$ but decreases rapidly above this limit. Consequently, it is motivated to define a threshold HCF strain range $\Delta \varepsilon_{H C F}^{T h}$ below which damage is not accelerated by the HCF cycling. The trend is even more apparent if the curves are normalised with reference to the $N_{f}$ values obtained below the HCF strain range threshold since all the curves will collapse into one curve, see Figure 10. This clearly demonstrates that $\Delta \varepsilon_{H C F}^{T h}$, and even 


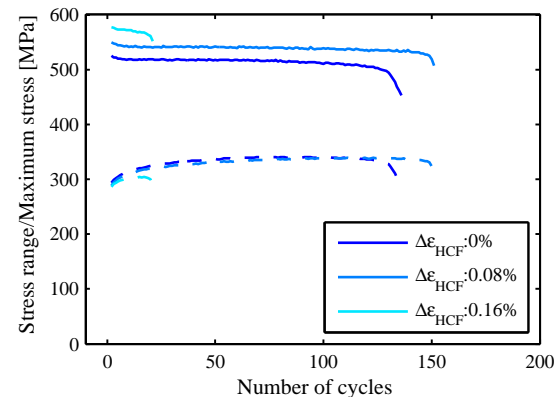

(a)

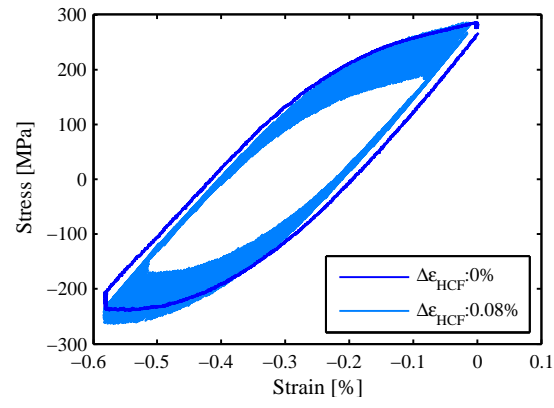

(b)

Figure 7: (a) The maximum stress and the stress range of each cycle during a TMF-HCF test, $100^{\circ} \mathrm{C}$ to $500^{\circ} \mathrm{C}$ and $\Delta \varepsilon_{M e c h}$ equal to $0.58 \%$, with different $\mathrm{HCF}$ strain loads and (b) a hysteresis loop of a TMF-HCF test with an applied HCF strain range of $0 \%$ and $0.08 \%$.

the normalised profile seen in Figure 10, are independent of the tested TMF parameters, i.e. $T_{M a x}$ and $\Delta \varepsilon_{M e c h}$, within the tested intervals. The value of $\Delta \varepsilon_{H C F}^{T h}$ is also in good agreement with the earlier mentioned threshold value of $0.06 \%$ found by Uihlein et al. [7].

A final remark is the apparent increase in fatigue life at low $\mathrm{HCF}$ strain ranges, $0.02-0.06 \%$. This could simply be due to scatter in the test data, however there are other reasons which could explain the trend. Examining Figure 7a closely, one can discern a small reduction in the maximum stress curve when an $\mathrm{HCF}$ strain range of $0.08 \%$ is applied, which likely could be responsible for slightly longer fatigue lives, given the condition of $\Delta \varepsilon_{H C F}$ being below the threshold.

\subsection{Prediction of the lifetime curves}

The reduction of the lifetime due to a superimposed HCF strain load as indicated in Figure 9a and 9b can be predicted by the following approach.

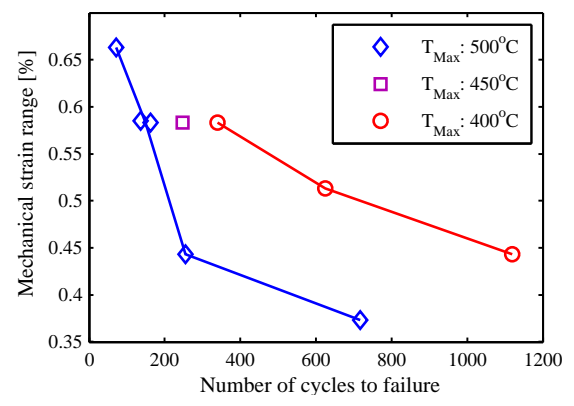

(a)

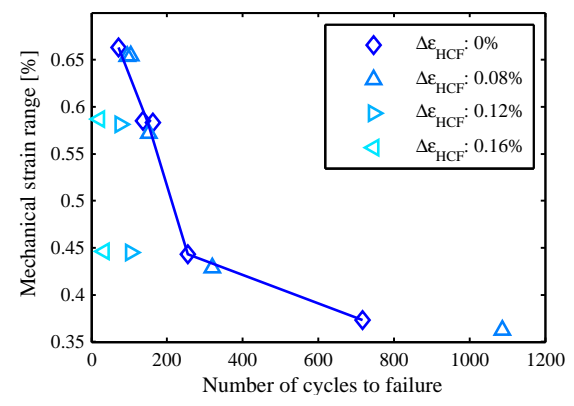

(b)

Figure 8: The strain life curve of (a) TMF tests with different $T_{M a x}$ and $\Delta \varepsilon_{M e c h}$ but without an HCF strain load and (b) TMF-HCF test at $T_{M a x}$ equal to $500^{\circ} \mathrm{C}$ for different $\Delta \varepsilon_{H C F}$. Each data point corresponds to one test. 


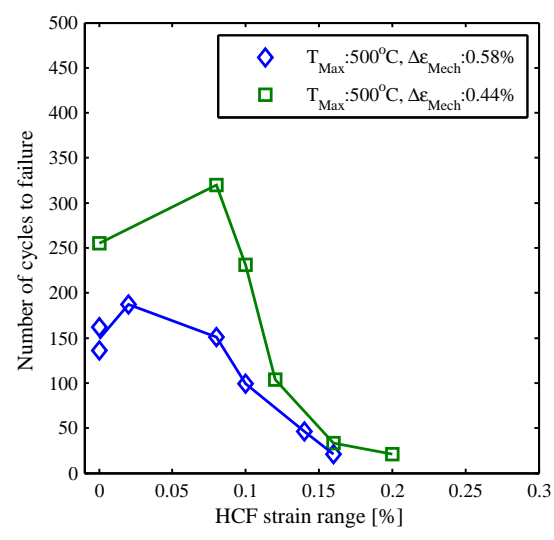

(a)

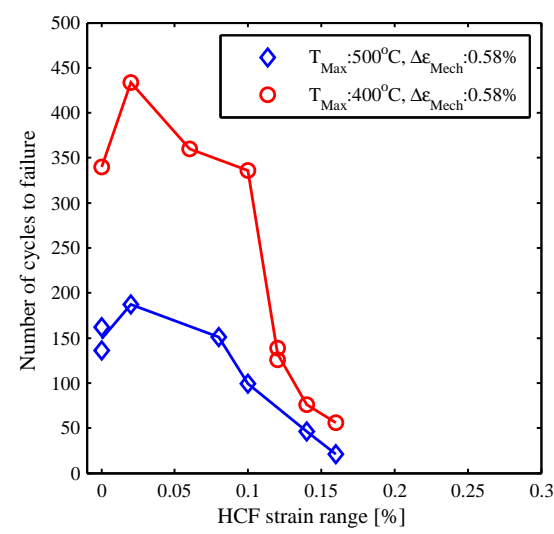

(b)

Figure 9: Alternative representations of lifetime curves with $N_{f}$ as a function of $\Delta \varepsilon_{H C F}$ applied in two different TMF test with (a) the same $T_{M a x}$ of $500^{\circ} \mathrm{C}$ and (b) the same $\Delta \varepsilon_{M e c h}$ of $0.58 \%$. Each data point corresponds to one test.

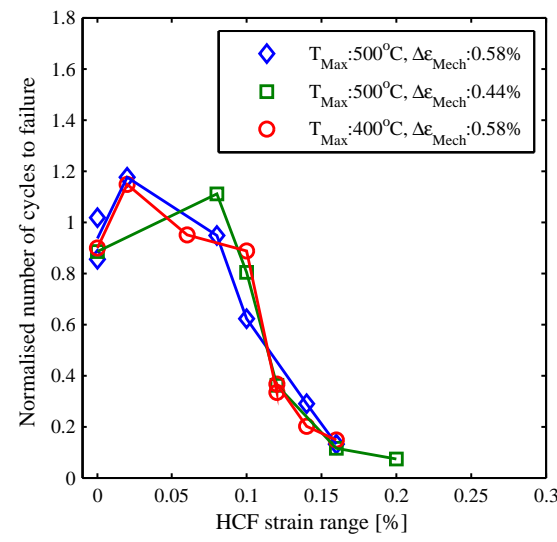

Figure 10: Normalised $\Delta \varepsilon_{H C F^{-}}-N_{f}$-curves with respect to the lifetimes obtained below the anticipated $\mathrm{HCF}$ strain range threshold of $0.08 \%$.

\subsubsection{Assumptions}

Initially, (i) it is hypothesised that the incremental crack extension $\Delta a$ due to an applied TMF cycle and the superimposed HCF cycles is separable as

$$
\Delta a=\Delta a_{T M F}+\sum_{i} \Delta a_{H C F}^{i}
$$

where $\Delta a_{T M F}$ is the crack extension due to one TMF cycle alone and $\Delta a_{H C F}^{i}$ are the crack extensions due to each HCF cycle $i$ elapsed during the TMF cycle. This approach has been employed by several others $[9,5]$ and is also supported by the previously mentioned observations by Beck et al. [8] that the sole effect of superimposed HCF loading is an accelerated crack growth rather than changes in the fatigue mechanism in spheroidal graphite iron. In addition, 
this assumption will imply the omission of overload phenomena and the resulting transient effects on the crack propagation rate, which is not unreasonable since the plastic deformation due to the HCF cycle is considered insignificant.

Secondly, (ii) guided by a previous investigation [13], and indications made earlier by others $[14,15,16]$, the crack propagation is believed to consist of the propagation of small and numerous microcracks, initiated at graphite tips and emanating into the matrix. The reason for initiation is rationalised by previous work investigating the graphite-matrix interaction in cast irons. The occurrence of graphite debonding [17] and internal graphite fracture [18] at low levels of strain during the first cycle is believed to result in starting points for fatigue crack propagation. Moreover, since it has been established that these microcracks are initiated and propagated homogeneously in the bulk and surface [13], the prediction model is aimed to account for the propagation of an average short crack required for the instability of the material as a whole. Consequently, the final crack length will correspond to the average microcrack length required for the onset of macrocrack formation through microcrack linking. This crack length will be denoted as $\bar{a}_{f}$ and is assumed to be in the order of the distance between two neighbouring graphite particles or the eutectic cell size; the bar indicating that it should be regarded as an averaged value. Accordingly, the initial average crack length is denoted $\bar{a}_{0}$ and is assumed to be in the order of the averaged size of the graphite particles.

For convenience, a Paris law is chosen as the propagation law of the microcracks given as

$$
\frac{d \bar{a}}{d N}=C[\Delta K(\Delta \sigma, \bar{a})]^{n}=C[\bar{Y} \Delta \sigma \sqrt{\pi \bar{a}}]^{n}
$$

where $\bar{a}$ is the average microcrack length, $N$ is the number of cycles, $\Delta K$ is the stress-intensity factor range of the average microcrack for a given stress range $\Delta \sigma, C$ and $n$ are the Paris law coefficient and exponent respectively. The variable $\bar{Y}$ is a dimensionless constant which depends on the crack geometry and mode of loading, and it is also considered an average here.

The Paris law requires a condition of small-scale yielding at the crack tip and that the concept of similitude is applicable, i.e. that the far field loading condition is comparable with the near tip conditions, which propagation of short crack often fails to fulfil [19]. For instance, one could argue that the matrix is subjected to a non-uniform stress field due to the stress concentrations induced by the graphite particles [20], thereby influencing the near tip conditions. However, Ghodrat et al. [16] managed successively to model fatigue crack propagation of notched CGI specimens in TMF testing using a Paris law.

The parameters $C$ and $n$ are most likely temperature dependent. To make things worse, the temperature is not constant during a TMF cycle. Thus, the $C$ and $n$ parameters do not depend on one temperature value, such as in isothermal LCF testing, but on the whole sequence of temperatures throughout the TMF cycle. Thus, the $C$ and $n$ parameters will depend on a particular temperature cycle.

In addition, the values of $C$ and $n$ will also differ when considering each $\mathrm{HCF}$ cycle. A particular HCF cycle will be applied at some moment during the TMF cycle and the $C$ and $n$ parameters will thus correspond to the current temperature of that instant. However, since the HCF cycles are rapid compared 
to the rate of change of the temperature, it is supposed that these parameters will depend on one instantaneous temperature only.

Finally, (iii) it is assumed, and verified experimentally in the next section, that the crack propagation is influenced by a crack opening phenomena which implies that there is some stress level, denoted $\sigma_{o p}$, beneath which the microcracks are closed and therefore not propagated. As a consequence, an HCF cycle applied at a stress level below the crack opening level in the TMF hysteresis loop will not propagate the microcracks and its contribution to the second term in Equation 3 will be zero.

Using the Paris law, Equation 4, the incremental crack extension due to one TMF cycle, i.e. the first term in Equation 3, can be rewritten as

$$
\Delta \bar{a}_{T M F}=C_{T M F}\left[\left(\sigma_{M a x}(N)-\sigma_{o p}\right) \bar{Y} \sqrt{\pi \bar{a}}\right]^{n_{T M F}} \Delta N
$$

where $C_{T M F}$ and $n_{T M F}$ are the Paris law coefficient and exponent respectively corresponding to the TMF temperature cycle, $\Delta N$ is one cycle increment, and $\sigma_{M a x}$ is the maximum stress in each cycle. As seen in Figure 5a, $\sigma_{M a x}$ is a function of the number of cycles $N$.

Regarding the crack propagation due to the HCF cycles, some of the HCF cycles are applied beneath the crack opening level while others are above, and a few HCF cycles only partially above. However, only the HCF cycles completely or partially above the the crack opening level will propagate the microcracks. Accordingly, one must keep track of the effective stress $\Delta \sigma_{H C F}^{E f f, i}$, i.e. the stress range extending above the crack opening stress level $\sigma_{o p}$, where $i$ is the index of each HCF cycle. Thus, the HCF contribution to the total incremental crack extension is written as

$$
\sum_{i} \Delta \bar{a}_{H C F}^{i}=\sum_{i} C_{H C F}^{i}\left[\Delta \sigma_{H C F}^{E f f, i} \cdot \bar{Y} \sqrt{\pi \bar{a}}\right]^{n_{H C F}^{i}} \Delta N^{i}
$$

where $C_{H C F}^{i}$ and $n_{H C F}^{i}$ are the Paris law coefficient and exponent respectively acting during the HCF cycle $i$ and $\Delta N^{i}$ are the HCF cycle increments. Adopting the (ii) argument, it is motivated to suppose that the crack opening stress level $\sigma_{o p}$ is constant with respect to the average crack length $\bar{a}$ since the microcracks are still assumed to be short at the point of macrocrack formation. Thus, the change in $\sigma_{o p}$ with $\bar{a}$ is neglected and will therefore be a constant value. Furthermore, the temperature dependence of $\sigma_{o p}$ is assumed to be negligible. The value of $\sigma_{o p}$ is discussed in the next section.

Combining Equation 3, 5 and 6 yields

$$
\left.\frac{d \bar{a}}{d N}=C_{T M F}\left(\sigma_{M a x}(N)-\sigma_{o p}\right) \bar{Y} \sqrt{\pi \bar{a}}\right]^{n_{T M F}}+\sum_{i} C_{H C F}^{i}\left[\Delta \sigma_{H C F}^{E f f, i} \cdot \bar{Y} \sqrt{\pi \bar{a}}\right]^{n_{H C F}^{i}}
$$

where the integer variables $N$ and $N^{i}$ have been replaced by continuous variables in order to perform the differentiation. In this limit, the infinitesimal increments $d N$ and $d N^{i}$ are regarded as equivalent. Equation 7 still needs a lot of information to be practically analysed, namely the values of all the $C$ and $n$ parameters. However, if the third assumption (iii) is valid, the microcracks are only open in the tensile part of the TMF cycle where the temperature is 
below $250^{\circ} \mathrm{C}$ due to the out-of-phase configuration. It is then motivated to suggest that the variations in $C$ and $n$ are small in this temperature range. Thus, in this approach all $C$ and $n$ parameters are taken as equal to $C_{T M F}$ and $n_{T M F}$ corresponding to the TMF cycle. It should be noted here that this is an engineering assumption which could be shown to be inaccurate, however the gain in simplicity and the good experimental fit to be seen in subsequent sections are clearly in the favour of this approach.

By these simplifications, Equation 7 becomes a separable differential equation. Collecting the $\bar{a}$ dependent part on the left side and the $N$ dependent part on the right, integration yields:

$$
\begin{aligned}
\int_{\bar{a}_{0}}^{\bar{a}_{f}} \frac{d \bar{a}}{[\bar{Y} \sqrt{\pi \bar{a}}]^{n_{T M F}}}= \\
\quad \int_{0}^{N_{f}} C_{T M F}\left(\left[\sigma_{M a x}(N)-\sigma_{o p}\right]^{n_{T M F}}+\sum_{i}\left[\Delta \sigma_{H C F}^{E f f, i}\right]^{n_{T M F}}\right) d N
\end{aligned}
$$

The left-hand side will be a function of the initial and final averaged crack lengths, however for a qualitative analysis it is enough to observe that the integral will be the same regardless of the test parameters, e.g. $T_{\text {Max }}$ or $\Delta \varepsilon_{M e c h}$. Therefore, the left-hand side integral will be regarded as a constant $I$ to ease the notation.

To ease the calculation, the second term in the integral can be simplified by only considering small elastic stresses, i.e. $\Delta \sigma_{H C F}=E \Delta \varepsilon_{H C F}$, and the average number of $\mathrm{HCF}$ cycles above the crack opening stress level, denoted $\rho$, as contributive to the summation. Consequently,

$$
C^{\prime} \int_{0}^{N_{f}}\left[\sigma_{M a x}(N)-\sigma_{o p}\right]^{n_{T M F}} d N=1-C^{\prime} \rho\left[E \Delta \varepsilon_{H C F}\right]^{n_{T M F}} N_{f}
$$

where $C^{\prime}$ equals $C_{T M F} / I$. The last mentioned simplification has been verified to influence the outcome negligibly by comparing the solutions given by Equation 8 and 9 for different values of $\Delta \varepsilon_{H C F}$.

\subsubsection{Determining the parameters}

The $C^{\prime}$ and $n_{T M F}$ parameters can be determined by the series of TMF tests without an HCF load. In this case, the right-hand side of Equation 9 is reduced to unity. Performing a series of TMF tests with the same temperature cycle but different total mechanical strain ranges will give the corresponding number of $\sigma_{\text {Max }}$ curves, such as in Figure 5a, and lifetimes $N_{f}$, which must fulfil this equation with the same set of $C^{\prime}$ and $n_{T M F}$. Thus, the $n_{T M F}$ exponent can be obtained by finding a $n_{T M F}$-value yielding the same value of the integral in

$$
\int_{0}^{N_{f}^{j}}\left[\sigma_{M a x}^{j}(N)-\sigma_{o p}\right]^{n_{T M F}} d N=\frac{1}{C^{\prime}}
$$

for each TMF test $j$ having the same temperature cycle. Accordingly, the optimal $C^{\prime}$ coefficient becomes the reciprocal of the integral averaged over $j$ and with the optimal $n_{T M F}$ value just obtained. The parameters will depend on the 
choice of $\sigma_{o p}$ which will be motivated in the next section. Calculated values of $C^{\prime}$ and $n_{T M F}$ using the TMF data presented in Figure 8a are given in Table 3.

It is noted that the Paris law exponent $n_{T M F}$ is much higher than the value found by Ghodrat et al. [16], however they tested notched specimens in contrast to the current study were smooth specimens have been tested. Regarding the former case, the life is governed by crack propagation of a dominant crack initiated at the notch, while in the latter, microcracks propagate throughout the specimen at multiple locations. In particular, the fact that many cracks propagate is believed to be the main reason for the remarkable high value of the Paris law exponent in the present study.

\subsubsection{Evaluation}

Having numerical values of $\sigma_{o p}, C^{\prime}$ and $n_{T M F}$, as well as a $\rho$ value and measured maximum stress curves $\sigma_{\text {Max }}(N)$, Equation 9 can be solved. The solution is most intuitively demonstrated graphically by plotting the left and right-hand side as functions of $N_{f}$, denoted $F_{T M F}\left(N_{f}\right)$ and $F_{H C F}\left(N_{f}\right)$ respectively, and evaluating the intersection, see Figure 11.

From this figure, one can clearly see the effect of changing $\Delta \varepsilon_{H C F}$. As this variable is increased, the negative slope of the right-hand side function will increase; moving the intersection point towards lower $N_{f}$ values. In addition, when $\Delta \varepsilon_{H C F}$ equals zero the lifetime will be determined by the solution in which the left-hand side function equals unity.

The solutions of $N_{f}$ using the parameters in Table 3 are plotted as a function of $\Delta \varepsilon_{H C F}$ in Figures 12a and 12b together with the experimental results from the previous section. The agreement is regarded as good considering that no additional fitting parameter is needed more than the parameters $C^{\prime}$ and $n_{T M F}$ obtained from pure TMF tests. However, it is observed that the model underestimates the fatigue life at high HCF strain ranges. This is believed to be due to the assumption of purely elastic HCF deformations, since it will imply overestimated stresses if the material yields at each HCF cycle. Concerning the

\begin{tabular}{|c|cc|}
\hline Temperature cycle & $C^{\prime}\left[M P a^{-n_{T M F}}\right]$ & $n_{T M F}[-]$ \\
\hline $100^{\circ} \mathrm{C}-400^{\circ} \mathrm{C}$ & $5.30 \cdot 10^{-36}$ & 12.98 \\
$100^{\circ} \mathrm{C}-500^{\circ} \mathrm{C}$ & $3.27 \cdot 10^{-35}$ & 12.49 \\
\hline
\end{tabular}

Table 3: Calculated parameters with $\sigma_{o p}=-25 M P a$.

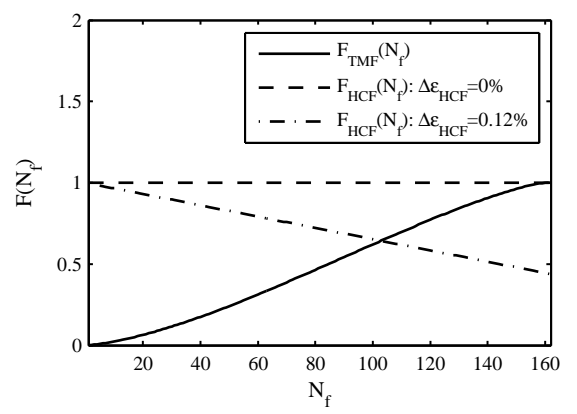

Figure 11: A representation of the graphical solution of Equation 9. 


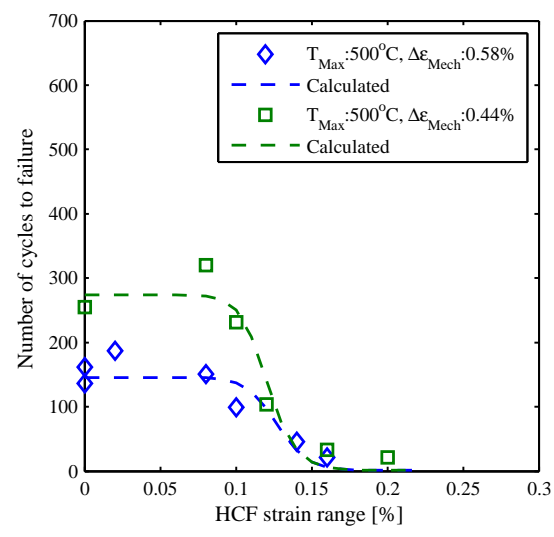

(a)

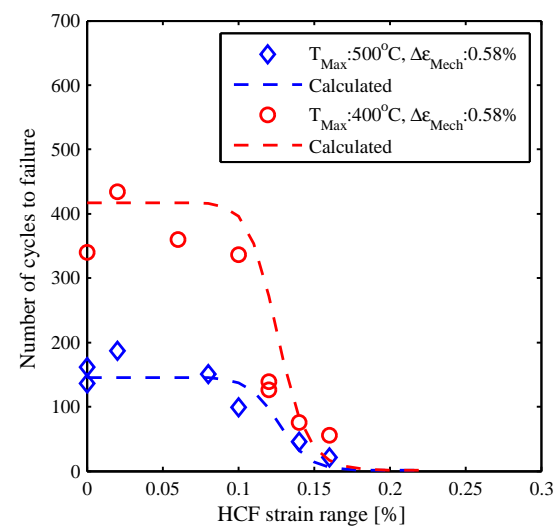

(b)

Figure 12: A comparison between calculated and experimental number of cycles to failure as a function of the HCF strain range for two different TMF cycles with (a) the same $T_{M a x}$ of $500^{\circ} \mathrm{C}$ and (b) the same $\Delta \varepsilon_{M e c h}$ of $0.58 \%$. Each data point corresponds to one test.

value of $\sigma_{o p}$ it can without any significant influence be supposed to be zero, as the obtained value of the next section is close to this.

\subsection{Experimental verification of crack opening}

Since the crack opening stress level $\sigma_{o p}$ was initially considered unknown, a small set of special TMF-HCF tests was conducted in order to make a reasonable estimation. All tests consisted of a common TMF cycle in an OP configuration, where the temperature was cycled between $100^{\circ} \mathrm{C}$ to $500^{\circ} \mathrm{C}$ and the total mechanical strain range was $0.58 \%$. An additional HCF strain range of $0.16 \%$ was applied, however only during the compressive part of the TMF cycle. Thus, a specific stress level was selected and varied, denoted $\sigma_{\text {on } / \text { off } f}$, above which no HCF cycles were applied, in order to observe the effect on the number of cycles to failure. The appearance of the stress variation for the third cycle of such a $\mathrm{HCF}$ on/off test is displayed in Figure 13a.

Figure 13b shows the outcome of a couple of tests where only the HCF cycle on/off stress level $\sigma_{\text {on } / \text { off }}$ is varied. It was experimentally difficult to select a specific stress for switching on and off the HCF cycles since the tests were strain controlled. Instead, specific strain levels were chosen corresponding to the targeted on/off stress, which led to some variation of it since the hysteresis loop is moving during the cycles, recall Figure 6. Consequently, the on/off stresses on the horizontal axis in Figure 13b are averaged values. Nonetheless, a clear decrease in the lifetime is observed when HCF cycles are allowed at high stress levels while unaffected when only applied at lower stress levels. Thus, it is clear that the HCF cycles during the compressive part of the TMF loop do not contribute to the damage propagation.

Using these results together with the deduced framework, Equation 8, a value of the crack opening stress level $\sigma_{o p}$ can be fitted. In this calculation, all HCF cycles with an effective stress range exceeding the crack opening level are considered in the summation in Equation 8, in contrast to the simplification made when obtaining Equation 9. Thus by varying the $\sigma_{o p}$ variable, which will 


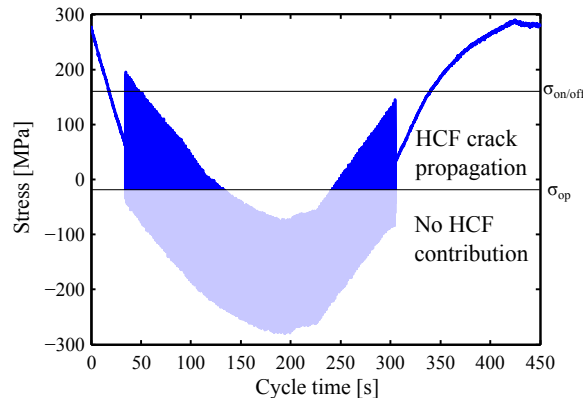

(a)

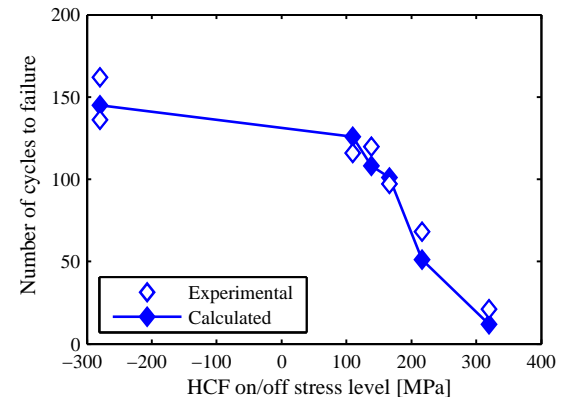

(b)

Figure 13: (a) The stress signal of a HCF on/off test, where the temperature is cycled between $100^{\circ} \mathrm{C}$ and $500^{\circ} \mathrm{C}$ and $\Delta \varepsilon_{M e c h}$ is equal to $0.58 \%$, with an $\mathrm{HCF}$ strain range of $0.16 \%$ and a stress level $\sigma_{\text {on } / \text { off }}$ above which the HCF cycles are turned off. The crack opening stress level $\sigma_{o p}$ is schematically added. (b) The number of cycles to failure $N_{f}$ as a function of the averaged $\mathrm{HCF}$ on/off stress level $\sigma_{\text {on/off }}$ obtained through experiments and calculations with $\sigma_{o p}=-25 M P a$. Each data point corresponds to one test.

influence Equation 8 and its fitting parameters, calculated lifetimes are fitted to the experimental data in Figure 13b. Consequently, an optimal value of $\sigma_{o p}$ was found as $-25 \mathrm{MPa}$ and the corresponding outcome is also shown in Figure $13 b$.

It should be noted that the obtained value of $\sigma_{o p}$ is not a direct measurement of the crack opening level but uses Equation 8 in order to make an estimate. On the other hand, the existence and its implications are undoubtedly demonstrated in Figure 13b. To make an explicit measurement, one should rather rely on crack propagation tests where the crack extension is measured as a function of the stress-intensity factor range $\Delta K$.

\section{Conclusions}

- The effect of adding a superimposed high-cycle fatigue (HCF) strain load upon a strain-controlled out-of-phase thermo-mechanical fatigue (TMF) test has been studied on a compacted graphite iron EN-GJV-400. From this, a threshold value of the HCF strain range has been identified, above which the fatigue life is severely reduced but unaffected beneath. In addition, it is demonstrated that the threshold appears to be the same regardless of the TMF cycle employed.

- It has been experimentally verified that crack propagation is affected by a crack opening level which is close to the zero stress level. Accordingly, it is verified that $\mathrm{HCF}$ cycles applied at a compressive stress do not propagate the fatigue cracks.

- The fatigue life reduction of a TMF tested specimen due to a superimposed HCF strain load was successively predicted using a Paris law model fitted to pure TMF data. 


\section{Acknowledgement}

The present study was financiered by Scania CV AB, the Swedish Governmental Agency for Innovation Systems (FFI - 2012 - 03625), and the Swedish Foundation for Strategic Research $(S M 12-0014)$. Agora Materiae and the Strategic Faculty Grant AFM (SFO-MAT-LiU\#2009-00971) at Linköping University are also acknowledged. Special thanks are also addressed to the project group at Scania for all their comments and feedback.

\section{References}

[1] M. Riedler, H. Leitner, B. Prillhofer, G. Winter, W. Eichlseder, Lifetime simulation of thermo-mechanically loaded components, Meccanica 42 (1) (2006) 47-59.

[2] J. J. Thomas, L. Verger, A. Bignonnet, E. Charkaluk, Thermomechanical design in the automotive industry, Fatigue and Fracture of Engineering Materials and Structures 27 (10) (2004) 887-895.

[3] S. Trampert, T. Gocmez, S. Pischinger, Thermomechanical Fatigue Life Prediction of Cylinder Heads in Combustion Engines, Journal of Engineering for Gas Turbines and Power 130 (012806) (2008) 1-10.

[4] T. Seifert, G. Maier, a. Uihlein, K.-H. Lang, H. Riedel, Mechanism-based thermomechanical fatigue life prediction of cast iron. Part II: Comparison of model predictions with experiments, International Journal of Fatigue 32 (8) (2010) 1368-1377.

[5] M. Metzger, B. Nieweg, C. Schweizer, T. Seifert, Lifetime prediction of cast iron materials under combined thermomechanical fatigue and high cycle fatigue loading using a mechanism-based model, International Journal of Fatigue 53 (2013) 58-66.

[6] T. Beck, D. Löhe, J. Luft, I. Henne, Damage mechanisms of cast Al-Si$\mathrm{Mg}$ alloys under superimposed thermal-mechanical fatigue and high-cycle fatigue loading, Materials Science and Engineering: A 468-470 (2007) 184192.

[7] A. Uihlein, K. Lang, D. Löhe, Lifetime Behavior at Superimposed ThermalMechanical and Mechanical Loading, in: Proceedings of the XIth International Congress and Exposition, 2008.

[8] T. Beck, K. Lang, D. Löhe, Interaction of thermally induced and mechanical fatigue, Transactions of The Indian Institute of Metals 63 (2-3) (2010) 195202.

[9] C. Schweizer, T. Seifert, B. Nieweg, P. von Hartrott, H. Riedel, Mechanisms and modelling of fatigue crack growth under combined low and high cycle fatigue loading, International Journal of Fatigue 33 (2) (2011) 194-202.

[10] H. Sehitoglu, Thermo-mechanical fatigue life prediction methods, in: ASTM Special Technical Publication, 1992, pp. 47-76. 
[11] P. Hähner, E. Affeldt, T. Beck, H. Klingelhöffer, M. Loveday, C. Rinaldi, Validated Code-of-Practice for Thermo-Mechanical Fatigue Testing (2006).

[12] T. Hammers, A. Uihlein, K. Lang, D. Löhe, Development of damage in cast iron during superimposed low frequency thermal-mechanical and higher frequency mechanical loading, in: Plasticity, Failure and Fatigue in Structural Materials-from Macro to Nano: Hael Mughrabi Honorary Symposium, New Orleans, 2008, pp. 253-258.

[13] V. Norman, P. Skoglund, J. Moverare, Damage Evolution in Compacted Graphite Iron during Thermo- Mechanical Fatigue Testing, in: Proceedings of the 10th Symposium on the Science and Processing of Cast Irons, INTEMA - UNMdP-CONICET, Mar del Plata, 2014.

[14] L. Haenny, G. Zambelli, Strain mechanisms in grey cast iron, Engineering Fracture Mechanics 18 (2) (1983) 377-387.

[15] H. Nisitani, S. Tanaka, Initiation and propagation of fatigue crack in cast iron, Transactions of the Japan Society of Mechanical Engineers, Part A 51 (465) (1985) 1442-1447.

[16] S. Ghodrat, A. Riemslag, M. Janssen, J. Sietsma, L. Kestens, Measurement and characterization of Thermo-Mechanical Fatigue in Compacted Graphite Iron, International Journal of Fatigue 48 (2013) 319-329.

[17] Z. He, S. Ji, G. Lin, Mechanical behaviour of graphite and its adjacent zone to matrix interface in cast iron, Acta metallurgia sinica 6 (3) (1993) $364-368$.

[18] R. Voigt, C. jun. Loper, Microstructural aspects of fracture in cast irons, in: Physical Metallurgy of Cast Iron IV, Proceedings 4th International Symposium, Tokyo, Japan, 1989, pp. 293-303.

[19] S. Suresh, Fatigue of materials, 1st Edition, Cambridge university press, Cambridge, 1991.

[20] T. Sjögren, P. E. Persson, P. Vomacka, Analysing the Deformation Behaviour of Compacted Graphite Cast Irons Using Digital Image Correlation Techniques, Key Engineering Materials 457 (2010) 470-475. 